\title{
Correspondence
}

\section{Cost containment in obstetric analgesia}

To the Editor:

Cheng et al. ${ }^{1}$ recently concluded that fentanyl provides similar analgesia at lower costs for labour analgesia compared to sufentanil. None of the currently available opioids possesses ideal pharmacokinetic properties for neuraxial use. Although limiting health care costs is warranted, care should be taken not to choose a less effective and potentially more harmful drug. In obstetric anesthesia, neonatal drug exposure is of equal importance, a point not discussed by the authors.

In an animal model, intrathecal fentanyl is more readily redistributed to the epidural space than sufentanil, ${ }^{2}$ so one may ask why not inject it epidurally in the first place? This is in part explained by its lower octanol: buffer distribution coefficient facilitating meningeal transfer. Accordingly, epidural fentanyl for labour analgesia results in higher maternal plasma levels and higher fetal opioid exposure with reduced neonatal neurobehavioural test scores compared with sufentanil. ${ }^{3}$ In addition, there is a trend toward superior analgesia with sufentanil. ${ }^{3}$ Since epidural analgesia is an important component of the combined spinalepidural technique, sufentanil for intrathecal injection would be taken from the same vial at no additional costs. In providing labour analgesia, anesthesiologists should reconsider choosing a more expensive technique with inherent risks and with no clinically significant benefit in properly designed trials, ${ }^{4}$ and then try to save these additional costs by opting for a potentially more harmful drug. We therefore propose to use epidural analgesia with sufentanil for labour, leaving the dura intact, and saving money.

Wiebke Gogarten $\mathrm{MD}$

Hartmut Buerkle MD PhD

Hugo Van Aken MD PhD

Münster, Germany

\section{References}

1 Cheng CJ, Sia AT, Lim EH, Loke GP, Tan HM. Either sufentanil or fentany, in addition to intrathecal bupivacaine, provide satisfactory early labour analgesia. Can J Anesth 2001; 48: 570-4.

2 Ummenhofer WC, Arends RH, Shen DD, Bernards CM. Comparative spinal distribution and clearance kinetics of intrathecally administered morphine, fentanyl, alfentanil, and sufentanil. Anesthesiology 2000; 92:

739-53.

3 Loftus JR, Hill H, Cohen SE. Placental transfer and neonatal effects of epidural sufentanil and fentanyl administered with bupivacaine during labor. Anesthesiology 1995; 83: 300-8.

4 Nickells JS, Vaughan DJ, Lillywhite NK, Loughnan B, Hasan M, Robinson PN. Speed of onset of regional analgesia in labour: a comparison of the epidural and spinal routes. Anaesthesia 2000; 55: 17-20.

\section{REPLY:}

We concur with Dr. Gogarten et al., that to harm or to exact a less than effective therapy to any patient for any "reason" at all (e.g., saving costs or for a vested interest on the part of the clinician) is totally unwarranted. On the other hand, one should not be overzealous in defending excessive expenditure, especially if it is for a dubious advantage. Our study showed that the clinical benefits and side effects were indistinguishable between fentanyl and sufentanil; as a corollary, the lower cost of fentanyl is an advantage. ${ }^{1}$

We believed it inappropriate to comment on the eventual neonatal well being as this would have been strongly influenced by a multitude of analgesia and non-analgesia related variables/exposures during the remaining intrapartum period. However, we did look at its surrogate, i.e., fetal heart rate tracing, and found no difference between fentanyl and sufentanil, albeit our study was probably underpowered here.

This is not a forum to discuss the veracity of extrapolating animal models to clinical anesthesia or the advantages/drawbacks of epidural fentanyl vs sufentanil; suffice it to say that over time, epidural infusion of both drugs does appear to produce analgesia by systemic uptake and redistribution. ${ }^{2,3}$ We therefore suggest a less dogmatic but more customized approach to labour epidural analgesia by using a low dose, multimodal (local anesthetic + lipophilic opioid $\pm_{2}$ agonist) technique and by considering combined spinal-epidural analgesia when indicated (e.g., late first stage of labour).

Alex T.H. Sia MB MMED

Christine J. Cheng MB FRCA

Evangeline H. Lim MB 
Genevieve P. Loke MB

Hsiao Ming Tan MB MMED

Singapore

References

1 Cheng CJC, Sia AT-H, Lim EH-L, Loke GP-Y, Tan

$H M$. Either sufentanil or fentanyl, in addition to intrathecal bupivacaine, provide satisfactory early labour analgesia. Can J Anesth 2001; 48: 570-4.

2 Miguel R, Barlow I, Morrell M, Scharf J, Sanusi D, Fu $E$. A prospective, randomized, double-blind comparison of epidural and intravenous sufentanil infusions. Anesthesiology 1994; 81: 346-52.

3 Ellis DJ, Millar WL, Reisner LS. A randomized doubleblind comparison of epidural versus intravenous fentanyl infusion for analgesia after cesarean section. Anesthesiology 1990; 72: 981-6.

\section{Fat embolism and postoperative coagulopathy}

To the Editor:

I was interested to read the article $^{l}$ and accompanying editorial $^{2}$ regarding fat embolism and postoperative coagulopathy.

Byrick points out that "even after cardiac arrest and resuscitation, such an impressive coagulopathy, leading to shock and death is exceedingly rare".

I recently reported a case of the fat embolism syndrome $^{3}$ in an 18-yr-old girl who had sustained a minimally displaced oblique fracture through the mid-shaft of her left tibia and fibula. In this case only very gentle, closed manipulation (performed within $24 \mathrm{hr}$ of the accident) was required to bring the bones to a suitable alignment. Nonetheless, she developed disseminated intravascular coagulation, multi-organ dysfunction syndrome, and refractory hypoxemia, dying approximately six days and a half after the initial injury.

I agree with Byrick's comment that 'intravascular fat may only be a marker for the intravasation of marrow products during cemented arthroplasty': this then begs the question why did such a common event ${ }^{4}$ have such devastating consequences in these two particular patients? Does it have anything to do with an abnormal and overwhelming inflammatory response? If so, who (and why) are certain people liable to such reactions?

In the case I described the only medical history of note was that of resolved but, at the time, debilitating myalgic encephalomyelitis. Was this another manifestation of an abnormally reactive immune/inflammatory system? If the immune system is involved how can we identify such patients, and such responses? As always "more research is needed".

C. Mark Harper BSC MB BS FRCA

Hertfordshire, UK

References

l Fallon KM, Fuller JG, Morely-Forster P. Fat embolization and fatal cardiac arrest during hip arthroplasty with methylmathacrylate. Can J Anesth 2001; 48:626-9.

2 Byrick RJ. Fat embolism and postoperative coagulopathy. Can J Anesth 2001; 48:618-21.

3 Harper CM. An unusual case of the fat embolism syndrome. Intensive Care Med 2000; 26: 819.

4 Koessler MJ, Fabiani R, Hamer H, Pitto RP. The clinical relevance of embolic events detected by transesophageal echocardiography during cemented hip arthroplasty: a randomized clinical trial. Anesth Analg 2001; 92: 49-55.

\section{Thoracoabdominal retroperitoneal lymph node dissection (RPLND) and bemodynamic instability in the postoperative period.}

To the Editor:

Thoracoabdominal retroperitoneal lymph node dissection is the most common and accepted method of treatment for patients with testicular cancer. This procedure involves extensive retroperitoneal dissection performed via a thoracoabdominal incision. All the lymph nodes, metastatic masses and lymphatic tissues are removed. In approximately $10 \%$ of cases the aorta and vena cava are removed and replaced with grafts. Coincidental removal of sympathetic ganglia and fibres during dissection is inevitable and may result in signs and symptoms of autonomic dysfunction in these patients, such as postural hypotension and tachycardia. $^{1,2}$ The signs and symptoms of autonomic dysfunction depend on the extent of the dissection, and such an extensive dissection is performed in $20-25 \%$ of the patients.

At the Norris Cancer Hospital we have done over 400 such cases and also managed them postoperatively in the intensive care unit. ${ }^{3}$ Our observation has been that the degree of hemodynamic instability (i.e., hypotension, tachycardia) directly correlates with the extent of the dissection. One-sided dissection results in ipsilateral signs of sympathectomy such as venous 